\title{
Einstein equations: exact solutions
}

\author{
Jiří Bičák \\ Institute of Theoretical Physics Charles University, Prague, Czech Republic \\ and \\ Albert Einstein Institute, Golm, Germany
}

\section{Introduction}

Even in a linear theory like Maxwell's electrodynamics, in which sufficiently general solutions of the field equations can be obtained, one needs a good sample, a useful kit, of explicit exact fields like the homogeneous field, the Coulomb monopole field, the dipole, and other simple solutions, in order to gain a physical intuition and understanding of the theory. In Einstein's general relativity, with its nonlinear field equations, the discoveries and analyses of various specific explicit solutions revealed most of the unforeseen features of the theory. Studies of special solutions stimulated questions relevant to more general situations, and even after the formulation of a conjecture about a general situation, newly discovered solutions can play a significant role in verifying or modifying the conjecture. The cosmic censorship conjecture assuming that "singularities forming in a realistic gravitational collapse are hidden inside horizons" is a good illustration.

Albert Einstein presented the final version of his gravitational field equations (EE) to the Prussian Academy in Berlin on November 18, 1915:

$$
R_{\mu \nu}-\frac{1}{2} g_{\mu \nu} R=\frac{8 \pi G}{c^{4}} T_{\mu \nu} .
$$

Here the spacetime metric tensor $g_{\mu \nu}\left(x^{\rho}\right), \mu, \nu, \rho, \ldots=$ $0,1,2,3$, determines the invariant line element $g=$ $g_{\mu \nu} d x^{\mu} d x^{\nu}$, and acts also as a dynamical variable describing the gravitational field; the Ricci tensor $R_{\mu \nu}=$ $g^{\rho \sigma} R_{\rho \mu \sigma \nu}$, where $g^{\mu \rho} g_{\rho \nu}=\delta_{\nu}^{\mu}$, is formed from the Riemann curvature tensor $R_{\rho \mu \sigma \nu}$; both depend nonlinearly on $g_{\alpha \beta}$ and $\partial_{\mu} g_{\alpha \beta}$, and linearly on $\partial_{\mu} \partial_{\nu} g_{\alpha \beta}$; the scalar curvature $R=g^{\mu \nu} R_{\mu \nu} . T_{\mu \nu}\left(x^{\rho}\right)$ is the energymomentum tensor of matter ("sources"); and Newton's gravitational constant $G$ and the velocity of light $c$ are fundamental constants. If not stated otherwise, we use the geometrized units in which $G=c=1$, and the same conventions as in 10], 14. For example, in the case of perfect fluid with density $\rho$, pressure $p$, and 4-velocity $U^{\mu}, T_{\mu \nu}=(\rho+p) U_{\mu} U_{\nu}+p g_{\mu \nu}$. To obtain a (local) solution of (1) in coordinate patch $\left\{x^{\rho}\right\}$ means to find "physically plausible" (i.e., complying with one of the positiveenergy conditions) functions $\rho\left(x^{\rho}\right), p\left(x^{\rho}\right), U_{\mu}\left(x^{\rho}\right)$, and metric $g_{\mu \nu}\left(x^{\rho}\right)$ satisfying (11). In vacuum $T_{\mu \nu}=0$ and
(11) implies $R_{\mu \nu}=0$.

In 1917, Einstein generalized (1) by adding a cosmological term $\Lambda g_{\mu \nu}(\Lambda=$ const $)$ :

$$
R_{\mu \nu}-\frac{1}{2} g_{\mu \nu} R+\Lambda g_{\mu \nu}=8 \pi T_{\mu \nu}
$$

A homogeneous and isotropic static solution of (2) (with metric (8), $k=+1, a=$ const), in which the "repulsive effect" of $\Lambda>0$ compensates the gravitational attraction of incoherent dust ("uniformly distributed galaxies") the Einstein static universe - marked the birth of modern cosmology. Although it is unstable and lost its observational relevance after the discovery of the expansion of the Universe in the late 1920s, in 2004 a "fine tuned" cosmological scenario was suggested according to which our universe starts asymptotically from an initial Einstein static state and later enters an inflationary era, followed by a standard expansion epoch [[see entry Cosmology: Mathematical Aspects]]. There are many other examples of "old" solutions which turned out to act as asymptotic states of more general classes of models.

\section{Invariant characterization and classification of the solutions}

\subsection{Algebraic classification}

The Riemann tensor can be decomposed as

$$
R_{\alpha \beta \gamma \delta}=C_{\alpha \beta \gamma \delta}+E_{\alpha \beta \gamma \delta}+G_{\alpha \beta \gamma \delta},
$$

where $E$ and $G$ are constructed from $R_{\alpha \beta}, R$ and $g_{\alpha \beta}$ (see, e.g., [13]); the Weyl conformal tensor $C_{\alpha \beta \gamma \delta}$ can be considered as the "characteristic of the pure gravitational field" since, at a given point, it cannot be determined in terms of the matter energy-momentum tensor $T_{\alpha \beta}$ (as $E$ and $G$ can using EE). Algebraic classification is based on a classification of the Weyl tensor. This is best formulated using 2-component spinors $\alpha_{A}(A=1,2)$, in terms of which any Weyl spinor $\Psi_{A B C D}$ determining $C_{\alpha \beta \gamma \delta}$ can be factorized:

$$
\Psi_{A B C D}=\alpha_{(A} \beta_{B} \gamma_{C} \delta_{D)},
$$

brackets denote symmetrization; each of the spinors determines a principal null direction, say, $k^{\alpha}=\alpha^{A} \bar{\alpha}^{A^{\prime}}$ 
[[see entry Spinors and Spin-coefficients]]. The PetrovPenrose classification is based on coincidences among these directions. A solution is of type $I$ (general case), $I I$, III, and $N$ ("null") if all null directions are different, or two, three, and all four coincide, respectively. It is of type $D$ ("degenerate") if there are two double null directions. The equivalent tensor equations are simplest for type $N$ :

$$
C_{\alpha \beta \gamma \delta} k^{\delta}=0, C_{\alpha \beta \gamma \delta} C^{\alpha \beta \gamma \delta}=0, C_{\alpha \beta \gamma \delta} C^{* \alpha \beta \gamma \delta}=0,
$$

where $C_{\alpha \beta \gamma \delta}^{*}=\frac{1}{2} \epsilon_{\alpha \beta \rho \sigma} C^{\rho \sigma}{ }_{\gamma \delta}, \epsilon$ is the Levi-Civita pseudotensor.

\subsection{Classification according to symme- tries}

Most of the available solutions have some exact continuous symmetries which preserve the metric. The corresponding group of motions is characterized by the number and properties of its Killing vectors $\xi^{\alpha}$ satisfying the Killing equation $\left(£_{\xi} g\right)_{\alpha \beta}=\xi_{\alpha ; \beta}+\xi_{\beta ; \alpha}=0(£$ is the Lie derivative) and by the nature (spacelike, timelike or null) of the group orbits. For example, axisymmetric, stationary fields possess two commuting Killing vectors, of which one is timelike. Orbits of the axial Killing vector are closed spacelike curves of finite length, which vanishes at the axis of symmetry. In cylindrical symmetry, there exist two spacelike commuting Killing vectors. In both cases, the vectors generate a 2-dimensional Abelian group. The 2-dimensional group orbits are timelike in the stationary case and spacelike in the cylindrical symmetry.

If a timelike $\xi^{\alpha}$ is hypersurface-orthogonal, $\xi_{\alpha}=\lambda \Phi_{, \alpha}$ for some scalar functions $\lambda, \Phi$, the spacetime is static. In coordinates with $\xi=\partial_{t}$, the metric is

$$
g=-e^{2 U} d t^{2}+e^{-2 U} \gamma_{i k} d x^{i} d x^{k},
$$

where $U, \gamma_{i k}$ do not depend on $t$. In vacuum, $U$ satisfies the potential equation $U_{: a}^{a}=0$, the covariant derivatives (denoted by :) are w.r.t. the 3-dimensional metric $\gamma_{i k}$. A classical result of Lichnerowicz states that if the vacuum metric is smooth everywhere and $U \rightarrow 0$ at infinity, the spacetime is flat (for refinements, see M.T. Anderson, 2000).

In cosmology, we are interested in groups whose regions of transitivity (points can be carried into one another by symmetry operations) are 3-dimensional spacelike hypersurfaces (homogeneous but anisotropic models of the Universe). The 3-dimensional simply transitive groups $G_{3}$ were classified by Bianchi in 1897 according to the possible distinct sets of structure constants but their importance in cosmology was discovered only in the 1950's. There are nine types: Bianchi I to Bianchi IX models. The line element of the Bianchi universes can be expressed in the form

$$
g=-d t^{2}+g_{a b}(t) \omega^{a} \omega^{b},
$$

where the time-independent 1-forms $\omega^{a}=E_{\alpha}^{a} d x^{\alpha}$ satisfy the relations $d \omega^{\alpha}=-\frac{1}{2} C_{b c}^{a} \omega^{b} \wedge \omega^{c}, d$ is the exterior derivative and $C_{b c}^{a}$ are the structure constants [[see entry Cosmology: Mathematical Aspects for more details]].

The standard Friedmann-Lemaitre-Robertson-Walker (FLRW) models admit in addition an isotropy group $\mathrm{SO}(3)$ at each point. They can be represented by the metric

$$
g=-d t^{2}+[a(t)]^{2}\left(\frac{d r^{2}}{1-k r^{2}}+r^{2}\left(d \theta^{2}+\sin ^{2} \theta d \varphi^{2}\right)\right),
$$

in which $a(t)$, the "expansion factor", is determined by matter via $\mathrm{EE}$, the curvature index $k=-1,0,+1$, the 3 -dimensional spaces $t=$ const have a constant curvature $K=k / a^{2} ; r \in[0,1]$ for closed $(k=+1)$ universe, $r \in[0, \infty)$ in open $(k=0,-1)$ universes (for another description, [[see entry Cosmology: Mathematical Aspects]]).

There are 4-dimensional spacetimes of constant curvature solving $\mathrm{EE}(2)$ with $T_{\mu \nu}=0$ : the Minkowski, de Sitter, and anti de Sitter spacetimes. They admit the same number (10) of independent Killing vectors, but interpretations of the corresponding symmetries differ for each spacetime.

If $\xi^{\alpha}$ satisfies $£_{\xi} g_{\alpha \beta}=2 \Phi g_{\alpha \beta}, \Phi=$ const, it is called a homothetic (Killing) vector. Solutions with proper homothetic motions $\Phi \neq 0$ are "self-similar". They cannot in general be asymptotically flat or spatially compact but can represent asymptotic states of more general solutions. In [13, a summary of solutions with proper homotheties is given; their role in cosmology is analyzed by J. Wainwright and G.F.R. Ellis (eds) 1996, and by A.A. Coley, 2003; for mathematical aspects of symmetries in general relativity, see G.S. Hall, 2004.

There are other schemes for invariant classification of exact solutions (reviewed in [13]): the algebraic classification of the Ricci tensor and energy-momentum tensor of matter; the existence and properties of preferred vector fields and corresponding congruences; local isometric embeddings into flat pseudo-Euclidian spaces, etc.

\section{Minkowski (M), de Sitter (dS), and anti de Sitter (AdS) space- times}

These metrics of constant (zero, positive, negative) curvature are the simplest solutions of (2) with $T_{\mu \nu}=0$ and $\Lambda=0, \Lambda>0, \Lambda<0$, respectively. The standard topology of $\mathrm{M}$ is $R^{4}$. The dS has the topology $R^{1} \times S^{3}$ and is best represented as a 4-dimensional hyperboloid $-v^{2}+w^{2}+x^{2}+y^{2}+z^{2}=(3 / \Lambda)$ in a 5 -dimensional flat space with metric $g=-d v^{2}+d w^{2}+d x^{2}+d y^{2}+d z^{2}$. The AdS has the topology $S^{1} \times R^{3}$; it is a 4 -dimensional hyperboloid $-v^{2}-w^{2}+x^{2}+y^{2}+z^{2}=-(3 / \Lambda), \Lambda<0$, in 
flat 5 -d space with signature $(-,-,+,+,+)$. By unwrapping the circle $S^{1}$ and considering the universal covering space, one gets rid of closed timelike lines.

These spacetimes are all conformally flat and can be conformally mapped into portions of the Einstein universe [[see entry Asymptotic Structure and Conformal Infinity]]. However, their conformal structure is globally different. In $\mathrm{M}$ one can go to infinity along timelike/null/spacelike geodesics and reach five qualitatively different sets of points: future/past timelike infinity $i^{ \pm}$, future/past null infinity $\mathcal{I}^{ \pm}$, and spacelike infinity $i^{0}$. In dS, there are only past and future conformal infinities $\mathcal{I}^{-}, \mathcal{I}^{+}$, both being spacelike (on the Einstein cylinder, the de Sitter spacetime is a "horizontal strip" with $\mathcal{I}^{+} / \mathcal{I}^{-}$as the "upper/lower circle"). The conformal infinity in AdS is timelike.

As a consequence of spacelike $\mathcal{I}^{ \pm}$in $\mathrm{dS}$, there exist both particle (cosmological) and event horizons for geodesic observers [7]. dS plays a (doubly) fundamental role in the present-day cosmology: it is an approximate model for inflationary paradigm near the Big Bang and it is also the asymptotic state (at $t \rightarrow \infty$ ) of cosmological models with a positive cosmological constant. Since recent observations indicate that $\Lambda>0$, it appears to describe the future state of our Universe. AdS has come recently to the fore due to the "holographic" conjecture [[see entry AdS/CFT Correspondence]].

Christodoulou and Klainermann, and Friedrich proved that $\mathrm{M}, \mathrm{dS}$, and AdS are stable with respect to general, nonlinear (though "weak") vacuum perturbationsresult not known for any other solution of EE [[see entry Stability of Minkowski Space]].

\section{Schwarzschild and Reissner- Nordström metrics}

These are spherically symmetric spacetimes - the $\mathrm{SO}_{3}$ rotation group acts on them as an isometry group with spacelike, 2-dimensional orbits. The metric can be brought into the form

$$
g=-e^{2 \nu} d t^{2}+e^{2 \lambda} d r^{2}+r^{2}\left(d \theta^{2}+\sin ^{2} \theta d \varphi^{2}\right),
$$

$\nu(t, r), \lambda(t, r)$ must be determined from EE. In vacuum, we are led uniquely to the Schwarzschild metric

$g=-\left(1-\frac{2 M}{r}\right) d t^{2}+\left(1-\frac{2 M}{r}\right)^{-1} d r^{2}+r^{2}\left(d \theta^{2}+\sin ^{2} \theta d \varphi^{2}\right)$,

where $M=$ const has to be interpreted as mass, as test particle orbits show. The spacetime is static at $r>2 M$, i.e., outside the Schwarzschild radius at $r=2 M$, and asymptotically $(r \rightarrow \infty)$ flat.

Metric (10) describes the exterior gravitational field of an arbitrary (static, oscillating, collapsing or expanding) spherically symmetric body (spherically symmetric gravitational waves do not exist). It is the most influential solution of EE. The essential tests of general relativityperihelion advance of Mercury, deflection of both optical and radio waves by the Sun, and signal retardation - are based on (10) or rather on its expansion in $M / r$. Space missions have been proposed that could lead to measurements of "post-post-Newtonian" effects [[see entry General Relativity: Experimental Tests and [10]]]. The full Schwarzschild metric is of importance in astrophysical processes involving compact stars and black holes.

Metric (10) describes the spacetime outside a spherical body collapsing through $r=2 M$ into a spherical black hole. In Fig. 1, the formation of an event horizon and trapped surfaces is indicated in ingoing Eddington-Finkelstein coordinates $(v, r, \theta, \varphi)$ where $v=$ $t+r+2 M \log (r / 2 M-1)$ so that $(v, \theta, \varphi)=$ const are ingoing radial null geodesics. The interior of the star is described by another metric (e.g., the Oppenheimer-Snyder collapsing dust solution - see below). The Kruskal extension of the Schwarzschild solution, its compactification, the concept of the bifurcate Killing horizon, etc., are analyzed in [[entry Stationary Black Holes]] and in [10, 7], [2.

The Reissner-Nordström solution describes the exterior gravitational and electromagnetic fields of a spherical body with mass $M$ and charge $Q$. The energymomentum tensor on the right-hand side of EE is that

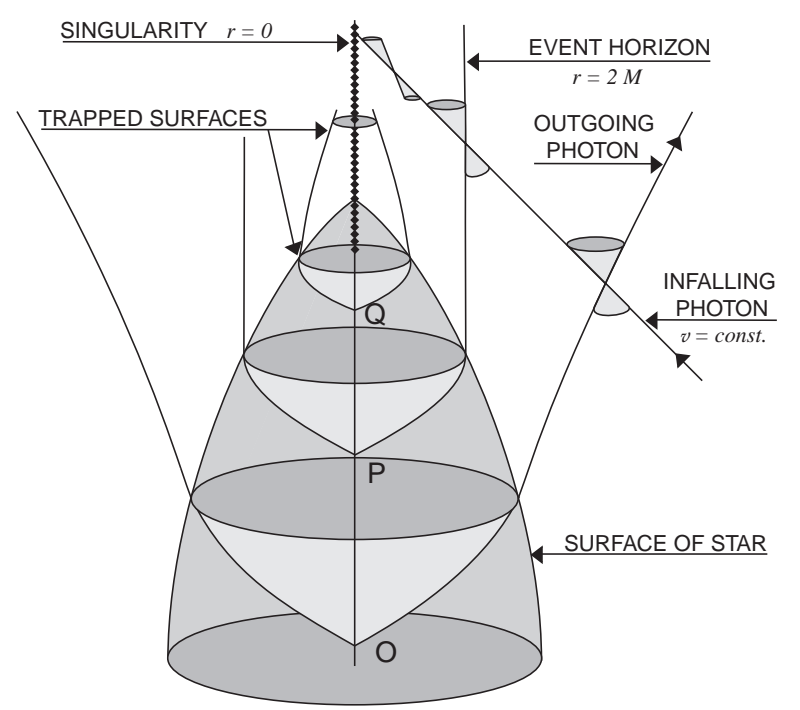

Figure 1 Gravitational collapse of a spherical star (the interior of the star is shaded). The light cones of three events, $O, P, Q$, at the center of the star, and of three events outside the star are illustrated. The event horizon, the trapped surfaces, and the singularity formed during the collapse are also shown. Although the singularity appears to lie along the direction of time, from the character of the light cone outside the star but inside the event horizon we can see that it has a spacelike character. 
of the electromagnetic field produced by the charge; the field satisfies the curved-space Maxwell equations. The metric reads

$$
\begin{aligned}
g= & -\left(1-\frac{2 M}{r}+\frac{Q^{2}}{r^{2}}\right) d t^{2}+ \\
& +\left(1-\frac{2 M}{r}+\frac{Q^{2}}{r^{2}}\right)^{-1} d r^{2}+r^{2}\left(d \theta^{2}+\sin ^{2} \theta d \varphi^{2}\right) .
\end{aligned}
$$

The analytic extension of the electrovacuum metric (11) is qualitatively different from the Kruskal extension of the Schwarzschild metric. In the case $Q^{2}>M^{2}$ there is a naked singularity (visible from $r \rightarrow \infty$ ) at $r=0$ where curvature invariants diverge. If $Q^{2}<M^{2}$, the metric describes a (generic) static charged black hole with two event horizons at $r=r_{ \pm}=M \pm\left(M^{2}-Q^{2}\right)^{1 / 2}$. The Killing vector $\partial / \partial t$ is null at the horizons, timelike at $r>r_{+}$and $r<r_{-}$, but spacelike between the horizons. The character of the extended spacetime is best seen in the compactified form, Fig. 2, in which worldlines of radial light rays are 45-degree lines. Again, two infinities (right and left, in regions I and III) arise (as in the Kruskal-Schwarzschild diagram-see [ [entry Stationary Black Holes]]), however, the maximally extended geometry consists of an infinite chain of asymptotically flat regions connected by "wormholes" between the singularities at $r=0$. In contrast to the Schwarzschild singularity, the singularities are timelike - they do not block the way to the future. The inner horizon $r=r_{-}$ represents a Cauchy horizon for a typical initial hypersurface like $\Sigma$ (Fig. 2): What is happening in regions $\mathrm{V}$ is in general influenced not only by data on $\Sigma$ but also at the singularities. The Cauchy horizon is unstable (for Refs., see [2] and recent work by M. Dafermos (2005)).

For $M^{2}=Q^{2}$ the two horizons coincide at $r_{+}=r_{-}=$ $M$. Metric (11) describes extreme Reissner-Nordström black holes. The horizon becomes degenerate and its surface gravity vanishes [[see entry Stationary Black Holes]]. Extreme black holes play a significant role in string theory 11.

\section{Stationary axisymmetric solu- tions}

Assume the existence of two commuting Killing vectorstimelike $\xi^{\alpha}$ and axial $\eta^{\alpha}\left(\xi^{\alpha} \xi_{\alpha}<0, \eta^{\alpha} \eta_{\alpha}>0\right)$, $\xi^{\alpha}$ normalized at (asymptotically flat) infinity, $\eta^{\alpha}$ at the rotation axis. They generate 2-dimensional orbits of the group $G_{2}$. Assume there exist 2-spaces orthogonal to these orbits. This is true in vacuum and also in case of electromagnetic fields or perfect fluids whose 4-current or 4 -velocity lies in the surfaces of transitivity of $G_{2}$ (e.g., toroidal magnetic fields are excluded). The metric can then be written in Weyl's coordinates $(t, \rho, \varphi, z)$

$$
g=-e^{2 U}(d t+A d \varphi)^{2}+e^{-2 U}\left[e^{2 k}\left(d \rho^{2}+d z^{2}\right)+\rho^{2} d \varphi^{2}\right]
$$

$U, k$, and $A$ are functions of $\rho, z$.

The most celebrated vacuum solution of the form (12) is the Kerr metric for which $U, k, A$ are ratios of simple polynomials in spheroidal coordinates (simply related to $(\rho, z))$. The Kerr solution is characterized by mass $M$ and specific angular momentum $a$. For $a^{2}>M^{2}$, it describes an asymptotically flat spacetime with a naked singularity. For $a^{2} \leq M^{2}$, it represents a rotating black hole that has two horizons which coalesce into a degenerate horizon for $a^{2}=M^{2}$-an extreme Kerr black hole. The two horizons are located at $r_{ \pm}=M \pm\left(M^{2}-a^{2}\right)^{\frac{1}{2}}(r$ being the Boyer-Lindquist coordinate-[ [see entry Stationary Black Holes]]). As with the Reissner-Nordström black hole, the singularity inside is timelike and the inner horizon is an (unstable) Cauchy horizon. The analytic extension of the Kerr metric resembles Fig. 2 (see [5], 7], 10-14 for details).

Thanks to the black-hole uniqueness theorems [[see entry Stationary Black Holes]], the Kerr metric is the unique solution describing all rotating black holes in vacuum. If the cosmic censorship conjecture holds, Kerr black holes represent the end states of gravitational collapse of astronomical objects with supercritical masses. According to prevalent views, they reside in the nuclei of most galaxies. Unlike with a spherical collapse, there are no exact solutions available which would represent the formation of a Kerr black hole. However, starting from metric (12) and identifying, e.g., $z=b=$ const and $z=-b$ (with the region $-b<z<b$ being cut off), one can construct thin material disks which are physically plausible and can be the sources of the Kerr metric even for $a^{2}>M^{2}$ (see [2] for details).

In a general case of metric (12), EE in vacuum imply the Ernst equation for a complex function $f$ of $\rho$ and $z$ :

$$
(\Re f)\left[f_{, \rho \rho}+f_{, z z}+\frac{1}{\rho} f_{, \rho}\right]=f_{, \rho}^{2}+f_{, z}^{2},
$$

or, equivalently, $(\Re f) \triangle f=(\nabla f)^{2}$, where $f=e^{2 U}+i b$, $U$ enters (12), and $b(\rho, z)$ is a "potential" for $A(\rho, z)$ : $A_{, \rho}=\rho e^{-4 U} b_{, z}, A_{, z}=-\rho e^{-4 U} b_{, \rho} ; k(\rho, z)$ in (12) can be determined from $U$ and $b$ by quadratures. Tomimatsu and Sato (TS) exploited symmetries of (13) to construct metrics generalizing the Kerr metric. Replacing $f$ by $\xi=(1-f) /(1+f)$, one finds that in case of the Kerr metric $\xi^{-1}$ is a linear function in the prolate spheroidal coordinates, whereas for TS solutions $\xi$ is a quotient of higher-order polynomials. A number of other solutions of Eq. (13) were found but they are of lower significance than the Kerr solution (cf. Ch. 20 in 13).

These solutions inspired "solution generating methods" in general relativity. The Ernst equation can be regarded as the integrability condition of a system of linear differential equations. The problem of solving such a system can be reformulated as the Riemann-Hilbert problem in complex function theory [[see entries RiemannHilbert problem, Integrable Systems]]. We refer to [13. 


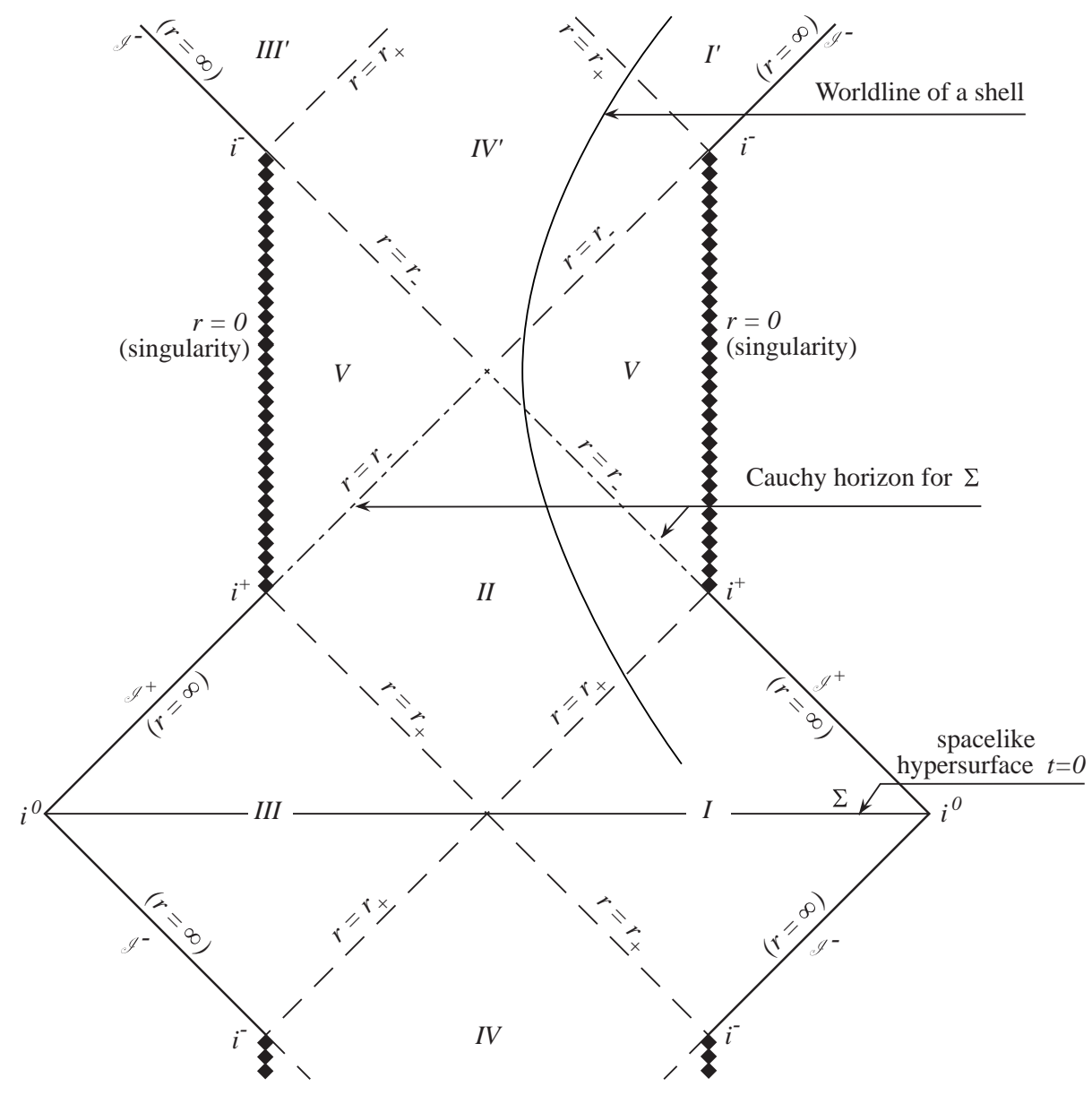

Figure 2 The compactified Reissner-Nordström spacetime representing a non-extreme black hole consists of an indefinite chain of asymptotic regions ("universes") connected by "wormholes" between timelike singularities. The worldline of a shell collapsing from "universe" $I$ and re-emerging in "universe" $I^{\prime}$ is indicated. The inner horizon at $r=r_{-}$is the Cauchy horizon for a spacelike hypersurface $\Sigma$. It is unstable and thus it will very likely prevent such a process.

and 1 where these techniques using Bäcklund transformations, inverse scattering method, etc. are also applied in the nonstationary context of two spacelike Killing vectors (waves, cosmology). In the stationary case, all asymptotically flat, stationary, axisymmetric vacuum solutions can, in principle, be generated. It is known how to generate fields with given values of multipole moments, though the required calculations are staggering. By solving the Riemann-Hilbert problem with appropriate boundary data, Neugebauer and Meinel constructed the exact solution representing a rigidly rotating thin disk of dust (cf. [13, 2]).

A subclass of metrics (12) is formed by static Weyl solutions with $A=b=0$. Eq. (13) then becomes the Laplace equation $\Delta U=0$. The non-linearity of EE enters only the equations for $k: k_{, \rho}=\rho\left(U_{, \rho}^{2}-U_{, z}^{2}\right), k_{, z}=$ $2 \rho U_{, \rho} U_{, z}$. The class contains some explicit solutions of interest: the "linear superposition" of collinear particles with string-like singularities between them which keep the system in static equilibrium; solutions representing external fields of counter-rotating disks, e.g., those which are "inspired" by galactic Newtonian potentials; disks around black holes and some other special solutions [13, [3], 2], 12.

There are solutions of the Einstein-Maxwell equations representing external fields of masses endowed with electric charges, magnetic dipole moments, etc. [13]. Best known is the Kerr-Newman metric characterized by parameters $M, a$, and charge $Q$. For $M^{2} \geq a^{2}+Q^{2}$ it describes a charged, rotating black hole. Owing to the rotation, the charged black hole produces also a magnetic field of a dipole type. All the black hole solutions can be generalized to include a non-vanishing $\Lambda$ (for various applications, see [12]). Other generalizations incorporate the so-called NUT (Newman-Unti-Tamburino) parameter (corresponding to a "gravomagnetic monopole") or an "external" magnetic/electric field or a parameter leading to "uniform" acceleration (see [13, 2]). Much interest has recently been paid to black-hole (and other) solutions with various types of gauge fields and to multidimensional solutions. Refs. [5] and [11] are two examples of good reviews. 


\section{Radiative solutions}

\subsection{Plane waves and their collisions}

The best known class are "plane-fronted gravitational waves with parallel rays" (pp-waves) which are defined by the condition that the spacetime admits a covariantly constant null vector field $k^{\alpha}: k_{\alpha ; \beta}=0$. In suitable null coordinates $u, v$ such that $k_{\alpha}=u_{, \alpha}, k^{\alpha}=(\partial / \partial v)^{\alpha}$, and complex $\zeta$ which spans the wave 2 -surfaces $u=$ const, $v=$ const with Euclidean geometry, the metric reads

$$
g=2 d \zeta d \bar{\zeta}-2 d u d v-2 H(u, \zeta, \bar{\zeta}) d u^{2},
$$

$H(u, \zeta, \bar{\zeta})$ is a real function. The vacuum EE imply $H_{, \zeta \bar{\zeta}}=0$ so that $2 H=f(u, \zeta)+\bar{f}(u, \bar{\zeta}), f$ is an arbitrary function of $u$, analytic in $\zeta$. The Weyl tensor satisfies equations (5) - the field is of type $N$ as is the field of plane electromagnetic waves. In the null tetrad $\left\{k^{\alpha}, l^{\alpha}, m^{\alpha}\right.$ (complex) $\}$ with $l^{\alpha} k_{\alpha}=-1, m^{\alpha} \bar{m}_{\alpha}=1$, all other products vanishing, the only nonzero projection of the Weyl tensor, $\Psi=C_{\alpha \beta \gamma \delta} l^{\alpha} \bar{m}^{\beta} l^{\gamma} m^{\delta}=H_{, \bar{\zeta} \bar{\zeta}}$, describes the transverse component of a wave propagating in the $k^{\alpha}$ direction. Writing $\Psi=\mathcal{A} e^{i \Theta}$, the real $\mathcal{A}>0$ is the amplitude of the wave, $\Theta$ describes polarization. Waves with $\Theta=$ const are called linearly polarized. Considering their effect on test particles one finds plane waves are transverse.

The simplest waves are homogeneous in the sense that $\Psi$ is constant along the wave surfaces. One gets $f(u, \zeta)=\frac{1}{2} \mathcal{A}(u) e^{i \Theta(u)} \zeta^{2}$. Instructive are sandwich waves, e.g., waves with a "square profile": $\mathcal{A}=0$ for $u<0$ and $u>a^{2}, \mathcal{A}=a^{-2}=$ const for $0 \leq u \leq a^{2}$. This example demonstrates, within exact theory, that the waves travel with the speed of light, produce relative accelerations of test particles, focus astigmatically generally propagating parallel rays, etc. The focusing effects have a remarkable consequence: there exists no global spacelike hypersurface on which initial data could be specified-plane wave spacetimes contain no global Cauchy hypersurface.

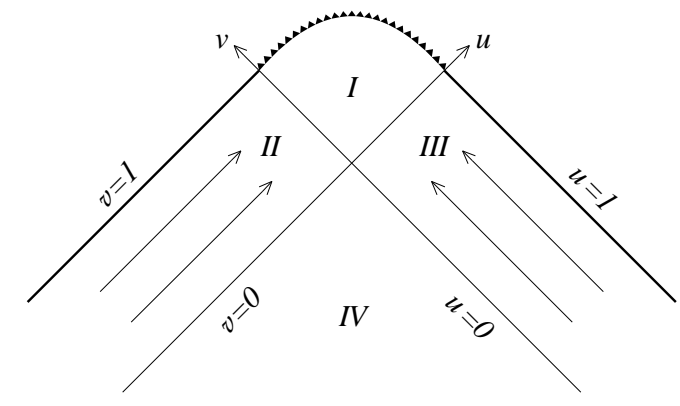

Figure 3 A spacetime diagram indicating a collision of two plane-fronted gravitational waves which come from regions $I I$ and $I I I$, collide in region $I$, and produce a spacelike singularity. Region $I V$ is flat.
Impulsive plane waves can be generated by boosting a "particle" at rest to the velocity of light by an appropriate limiting procedure. The ultrarelativistic limit of, e.g., the Schwarzschild metric (the so-called AichelburgSexl solution) can be employed as a "limiting incoming state" in black hole encounters (cf. monograph by P.D. d'Eath, 1996). Plane-fronted waves have been used in quantum field theory. For a review of exact impulsive waves, see [12].

A collision of plane waves represents an exceptional situation of nonlinear wave interactions which can be analyzed exactly. Fig. 3 illustrates a typical case in which the collision produces a spacelike singularity. The initial value problem with data given at $v=0$ and $u=0$ can be formulated in terms of the equivalent matrix RiemannHilbert problem [[see entry Riemann-Hilbert problem]]; it is related to the hyperbolic counterpart of the Ernst equation (13). For reviews, see [6], 13], and [2].

\subsection{Cylindrical waves}

Discovered by G. Beck in 1925 and known today as the Einstein-Rosen waves (1937), these vacuum solutions helped to clarify a number of issues, such as energy loss due to the waves, asymptotic structure of radiative spacetimes, dispersion of waves, quasi-local mass-energy, cosmic censorship conjecture, or quantum gravity in the context of midisuperspaces (see [2, 1]).

In the metric

$$
g=e^{2(\gamma-\psi)}\left(-d t^{2}+d \rho^{2}\right)+e^{2 \psi} d z^{2}+\rho^{2} e^{-2 \psi} d \varphi^{2},
$$

$\psi(t, \rho)$ satisfies the flat-space wave equation and $\gamma(\rho, t)$ is given in terms of $\psi$ by quadratures. Admitting a "cross term" $\sim \omega(t, \rho) d z d \phi$, one acquires a second degree of freedom (a second polarization) which makes all field equations nonlinear.

\subsection{Boost-rotation symmetric space- times}

These are the only explicit solutions available which are radiative and represent the field of finite sources. Fig. 4 shows two particles uniformly accelerated in opposite directions. In the space diagram (left), the "string" connecting the particles is the "cause" of the acceleration. In "Cartesian-type" coordinates and the $z$-axis chosen as the symmetry axis, the boost Killing vector has a flatspace form, $\zeta=z(\partial / \partial t)+t(\partial / \partial z)$, the same is true for the axial Killing vector. The metric contains two functions of variables $\rho^{2} \equiv x^{2}+y^{2}$ and $\beta^{2} \equiv z^{2}-t^{2}$. One satisfies the flat-space wave equation, the other is determined by quadratures.

The unique role of these solutions is exhibited by the theorem which states that in axially symmetric, locally asymptotically flat spacetimes in the sense that a null infinity [[see entry Asymptotic structure and conformal in- 

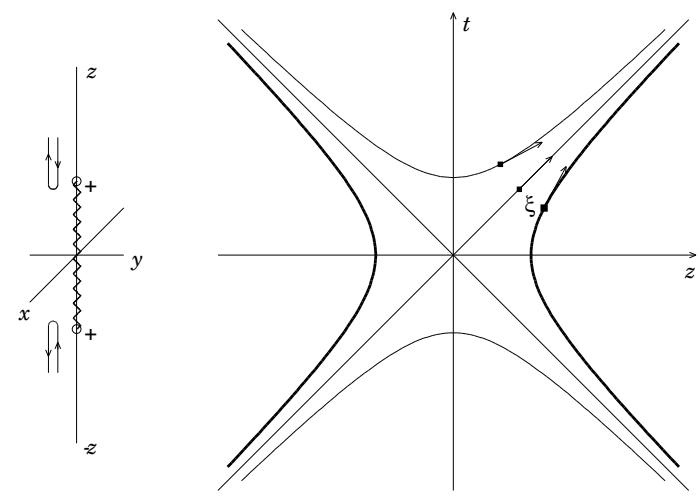

Figure 4 Two particles uniformly accelerated in opposite directions. Orbits of the boost Killing vector (thinner hyperbolas) are spacelike in the region $t^{2}>z^{2}$.

finity]] exists but not necessarily globally, the only additional symmetry that does not exclude gravitational radiation is the boost symmetry. Various radiation characteristics can be expressed explicitly in these spacetimes. They have been used as tests in numerical relativity and approximation methods. The best known example is the C-metric (representing accelerating black holes, in general charged and rotating, and admitting $\Lambda$ ) - see [4, [2], [13, 12 .

\subsection{Robinson-Trautman solutions}

These solutions are algebraically special but in general they do not possess any symmetry. They are governed by a function $P(u, \zeta, \bar{\zeta}) \quad(u$ is the retarded time, $\zeta$ a complex spatial coordinate) which satisfies a 4 th-order nonlinear parabolic differential equation. Studies by Chruściel and others have shown that RT solutions of Petrov type II exist globally for all positive "times" $u$ and converge asymptotically to a Schwarzschild metric, though the extension across the "Schwarzschild-like" horizon can only be made with a finite degree of smoothness. Generalization to the cases with $\Lambda>0$ gives explicit models supporting the cosmic no-hair conjecture (an exponentially fast approach to the de Sitter spacetime) under the presence of gravitational waves. See [4, 2], 13].

\section{$7 \quad$ Material sources}

Finding physically sound material sources in an analytic form even for some simple vacuum metrics remains an open problem. Nevertheless, there are solutions representing regions of spacetimes filled with matter which are of considerable interest.

One of the simplest, the spherically symmetric
Schwarzschild interior solution with incompressible fluid as its source, represents "a star" of uniform density, $\rho=\rho_{0}=$ const:

$$
\begin{aligned}
g= & -\left[\frac{3}{2} \sqrt{1-A R^{2}}-\frac{1}{2} \sqrt{1-A r^{2}}\right]^{2} d t^{2}+ \\
& +\frac{d r^{2}}{1-A r^{2}}+r^{2}\left(d \theta^{2}+\sin ^{2} \theta d \varphi^{2}\right),
\end{aligned}
$$

$A=8 \pi \rho_{0} / 3=$ const, $R$ is the radius of the star.

The equation of hydrostatic equilibrium yields pressure inside the star:

$$
8 \pi p=2 A \frac{\sqrt{1-A r^{2}}-\sqrt{1-A R^{2}}}{3 \sqrt{1-A R^{2}}-\sqrt{1-A r^{2}}} .
$$

Solution (16) can be matched at $r=R$, where $p=0$, to the exterior vacuum Schwarzschild solution (10) if the Schwarzschild mass $M=\frac{1}{2} A R^{3}$. Although "incompressible fluid" implies an infinite speed of sound, the above solution provides an instructive model of relativistic hydrostatics. A Newtonian star of uniform density can have an arbitrarily large radius $R=\sqrt{3 p_{c} / 2 \pi \rho_{0}^{2}}$ and mass $M=\left(p_{c} / \rho_{0}^{2}\right) \sqrt{6 p_{c} / \pi}, p_{c}$ is the central pressure. However, (17) implies that (i) $M$ and $R$ satisfy the inequality $2 M / R \leq 8 / 9$, (ii) equality is reached as $p_{c}$ becomes infinite and $R$ and $M$ attain their limiting values $R_{\lim }=\left(3 \pi \rho_{0}\right)^{-\frac{1}{2}}=(9 / 4) M_{\lim }$. For a density typical in neutron stars, $\rho_{0}=10^{15} \mathrm{~g} \mathrm{~cm}^{-3}$, we get $M_{\mathrm{lim}} \doteq 3.96 M_{\odot}$ $\left(M_{\odot}\right.$ solar mass) - even this simple model shows that in Einstein's theory neutron stars can only be a few solar masses. In addition, one can prove that the "Buchdahl's inequality" $2 M / R \leq 8 / 9$ is valid for an arbitrary equation of state $p=p(\rho)$. Only a limited mass can thus be contained within a given radius in general relativity. The gravitational redshift $z=(1-2 M / R)^{-\frac{1}{2}}-1$ from the surface of a static star cannot be higher than 2 .

Many other explicit static perfect fluid solutions are known (we refer to 13 for a list), however, none of them can be considered as really "physical". Recently, the dynamical systems approach to relativistic spherically symmetric static perfect fluid models was developed which gives qualititative characteristics of masses and radii (cf. work by C. Uggla et al.).

The most significant nonstatic spacetime describing a bounded region of matter and its external field is undoubtedly the Oppenheimer-Snyder model of gravitational collapse of a spherical star of uniform density and zero pressure (a "ball of dust"). The model does not represent any new (local) solution: the interior of the star is described by a part of a dust-filled FLRW universe (cf. (8)), the external region by the Schwarzschild vacuum metric (cf. Eq. (10), Fig. 1).

Since Vaidya's discovery of a "radiating Schwarzschild metric", null dust ("pure radiation field") has been widely used as a simple matter source. Its energymomentum tensor, $T_{\alpha \beta}=\varrho k_{\alpha} k_{\beta}$, where $k_{\alpha} k^{\alpha}=0$, may 
be interpreted as an incoherent superposition of waves with random phases and polarizations moving in a single direction, or as "lightlike particles" (photons, neutrinos, gravitons) that move along $k^{\alpha}$. The Vaidya metric describing spherical implosion of null dust implies that in case of a "gentle" inflow of the dust, a naked singularity forms. This is relevant in the context of the cosmic censorship conjecture (cf., e.g., [8]).

\section{Cosmological models}

There exist important generalizations of the standard FLRW models other than the above-mentioned Bianchi models, in particularly those that maintain spherical symmetry but do not require homogeneity. The best known are the Lemaitre-Tolman-Bondi models of inhomogeneous universes of pure dust, the density of which may vary 9 . (J. Gair recently generalized these models by including anisotropic pressure and null dust.)

Other explicit cosmological models of principal interest involve, e.g., the Gödel universe - a homogeneous, stationary spacetime with $\Lambda<0$ and incoherent rotating matter in which there exist closed timelike curves through every point; the Kantowski-Sachs solutionspossessing homogeneous spacelike hypersurfaces but (in contrast to the Bianchi models) admitting no simplytransitive $\mathrm{G}_{3}$; and vacuum Gowdy models ("generalized Einstein-Rosen waves") admitting $\mathrm{G}_{2}$ with compact 2tori as its group orbits and representing cosmological models closed by gravitational waves. See [[entry Cosmology: Mathematical Aspects]] and Refs. [13, [1, [2, 7], 9].

\section{See also:}

General Relativity: Overview. Stationary Black Holes. Asymptotic Structure and Conformal Infinity. Newtonian Limit of General Relativity. Cosmology: Mathematical Aspects. Spinors and Spin-Coefficients. Spacetime Topology, Causal Structure and Singularities. Lie Groups and Lie Algebras. General Relativity: Experimental Tests.

\section{Suggestions for further reading:}

$[1-14]$

\section{References}

[1] Belinski V and Verdaguer E (2001), Gravitational Solitons. Cambridge University Press, Cambridge.

[2] Bičák J (2000), Selected Solutions of Einstein's Field Equations: Their Role in General Relativ- ity and Astrophysics, in Einstein's Field Equations and Their Physical Implications, ed. B. G. Schmidt. Lecture Notes in Physics 540, Springer Verlag, Berlin-Heidelberg-New York, 1-126; see also gr-qc/0004016

[3] Bonnor WB (1992), Physical Interpretation of Vacuum Solutions of Einstein's Equations. Part I. Timeindependent Solutions. General Relativity and Gravitation 24, 551-574.

[4] Bonnor WB, Griffiths JB, and MacCallum MAH (1994), Physical Interpretation of Vacuum Solutions of Einstein's Equations. Part II. Time-dependent Solutions. General Relativity and Gravitation 26, 687-729.

[5] Frolov VP and Novikov ID (1998), Black Hole Physics. Kluwer Academic Publishers, DordrechtBoston-London.

[6] Griffiths JB (1991), Colliding Plane Waves in General Relativity. Oxford University Press, Oxford.

[7] Hawking SW and Ellis GFR (1973), The Large Scale Structure of Space-Time. Cambridge University Press, Cambridge.

[8] Joshi PS (1993), Global Aspects in Gravitation and Cosmology. Clarendon Press, Oxford.

[9] Krasiński A (1997), Inhomogeneous Cosmological Models. Cambridge University Press, Cambridge.

[10] Misner C, Thorne KS, and Wheeler JA (1973), Gravitation. W. H. Freeman and Co., San Francisco.

[11] Ortín T (2004), Gravity and Strings. Cambridge University Press, Cambridge.

[12] Semerák O, Podolský J, and Žofka M (eds.) (2002), Gravitation: Following the Prague Inpiration. World Scientific, Singapore-London.

[13] Stephani H, Kramer D, MacCallum MAH, Hoenselaers C, and Herlt E (2003), Exact Solutions of Einstein's Field Equations - Second Edition. Cambridge University Press, Cambridge.

[14] Wald RM (1984), General Relativity. The University of Chicago Press, Chicago. 